\title{
LA IMPORTANCIA DE LA DEFINICIÓN \\ LEXICOGRÁFICA PARA LA ADQUISICIÓN \\ EXITOSA DE VOCABULARIO EN ESTUDIANTES \\ DE ENSEÑANZA MEDIA. UNA MIRADA DESDE EL PANORAMA EDUCACIONAL CHILENO
}

\author{
THE IMPORTANCE OF THE LEXICOGRAPHICAL DEFINITION \\ FOR THE SUCCESSFUL ACQUISITION OF VOCABULARY IN \\ STUDENTS OF AVERAGE EDUCATION. A LOOK FROM THE \\ EDUCATIONAL CHILEAN PANORAMA
}

\author{
NATALIA VILLARROEL \\ Universidad de Chile, Santiago, Chile. \\ n.villarroeltorres@gmail.com \\ SUSANA SERRA \\ Universidad de Chile, Santiago, Chile. \\ sserra@uchile.cl
}

\section{RESUMEN}

La presente investigación analiza la capacidad "codificadora” presentada por un grupo de 42 estudiantes chilenos de enseñanza media, a partir de la aplicación de un modelo de definición lexicográfica basado en los presupuestos de la léxico-sintaxis. Desde una perspectiva proyeccionista del léxico (Bosque, 2004; Alonso, 2007 y Serra, 2013), se quiso verificar si tal modelo, en comparación con los tradicionales, particularmente el del DLE (Diccionario de la Lengua Española (2014)), entregaba información adecuada y exacta sobre los definidos y si esto propiciaba, o no, que nuestros informantes realizaran una correcta codificación de los significados de las palabras sometidas a examen. Los resultados del estudio revelaron que, tanto la carencia de restricciones léxicas adecuadas en los contornos de las definiciones, así como la ausencia de estos mismos, fueron los factores que propiciaron la construcción de oraciones agramaticales, las que se presentaron mayormente en el caso de las definiciones ofrecidas por el DLE (2014).

Palabras clave: Definición lexicográfica, perspectiva proyeccionista del léxico, contorno, restricción léxica, codificación. 


\begin{abstract}
In this study we analyze the ability of a group of 42 chilean students to encode information from the application of a lexicographical definition model based on the budgets of the lexicon-syntax. From a projectionist perspective (Bosque, 2004; Alonso, 2007 and Serra, 2013), we wanted to verify whether such a model, compared with the traditional one, particularly DLE (Diccionario de la Lengua Española) (2014), provide sufficient and necessary information in order to promote a correct coding of the meanings of the words under consideration. The study results showed that both the lack of appropriate contours and the absence of lexical constraints, were the factors that led to the construction of ungrammatical sentences, which occurred mostly in the case of the definitions provided by the DLE (2014).
\end{abstract}

Keywords: Lexicographical definition, projectionist lexical perspective, contour, lexical restriction, coding.

Recibido: 06.10.2017. Aceptado: 05.05.2018.

\title{
1. INTRODUCCIÓN
}

Dara comunicarse, los seres humanos ponen en práctica dos procesos cognitivos de gran importancia. Nos referimos a los procesos de codificación y de descodificación, los cuales no solamente hacen posible un acto tan cotidiano como sostener un diálogo (hablar, escuchar y comprender), sino también elaborar e interpretar mensajes más complejos, como los que recibimos a través de la prensa y el discurso escrito en general.

En la etapa de escolarización, los niños se ven enfrentados de manera sistemática a los libros de texto, a las gramáticas y a los diccionarios de lengua, recursos que contribuyen significativamente a la adquisición y desarrollo de estas capacidades humanas, consideradas tan importantes para el desarrollo del individuo moderno -tanto así, que en la actualidad son muchos los países que han creado instrumentos para medirlas.

En Chile, evaluaciones como el SIMCE (Sistema de Medición de la Calidad de la Educación) y la PSU (Prueba de Selección Universitaria) son algunas de las instancias que buscan constatar, entre otras cosas, cómo la población chilena está logrando estos dos niveles de competencia lingüística. Por otro lado, instituciones como Educarchile, OrientaChile y DEMRE ${ }^{1}$ (que se dedican a crear y evaluar instrumentos de enseñanza), han sido categóricas al afirmar que en toda la batería de pruebas de la PSU (lenguaje, matemáticas, historia y ciencias), los errores tienen lugar comúnmente a causa del déficit en comprensión de lectura. A lo anterior

${ }^{1}$ Sitio DEMRE www.demre.cl 
se suma el hecho de que la PSU de Lenguaje, además del nivel de comprensión lectora, se propone medir la competencia léxica de los alumnos, para lo cual ha incorporado el ítem de "vocabulario o léxico contextual”, que ha resultado ser especialmente complejo, según evidencian los datos. Concretamente, solo un $15 \%{ }^{2}$ de los evaluados contesta según las expectativas, mientras que el porcentaje restante cae en las trampas de la prueba, o bien, opta por no responder.

En virtud de lo expuesto, surge la interrogante de si las herramientas empleadas en la alfabetización de la población escolar chilena (libros de texto, manuales de gramática, diccionarios de lengua, etc.) son lo suficientemente adecuadas, o no lo son en la medida deseable, para el fin que están destinadas: esto es, si propician debidamente, o no, el desarrollo de las capacidades de codificación y descodificación en lengua materna de nuestros educandos. En otras palabras, a través del siguiente estudio se pretende poner en relación las prácticas educativas chilenas con el alcance o nivel de logros de los estudiantes en este sentido.

Considerando que instituciones y organismos educacionales como Educarchile, OrientaChile, DEMRE, etc., ya se ocupan de evaluar la capacidad de descodificación mediante instrumentos diversos de medición, nosotros nos hemos propuesto evaluar el otro aspecto comprometido en el proceso comunicativo, esto es, el de codificación. Así, valiéndonos de los resultados poco halagüeños que arrojó la PSU de Lenguaje correspondiente al año 2015 en el ítem de "vocabulario contextual", veremos si el proceso inverso en torno al léxico -el que atañe al "uso" de las piezas léxicas en un contexto definido-, también resulta ser un aspecto problemático para los jóvenes chilenos.

En concreto, lo que se quiere probar es si, al ser deficiente la capacidad descodificadora de los alumnos de enseñanza media, tal como señalan los datos, necesariamente se ve afectado el proceso de codificación de la información con el que se encuentra estrechamente relacionado para el logro de un proceso de comunicación exitoso. Para estos efectos, nos serviremos del instrumento de apoyo por excelencia para la enseñanza de idiomas (materno o extranjero) en la escuela: el diccionario, cuya finalidad es precisamente servir de guía en el proceso de adquisición del léxico por parte de los usuarios de una lengua.

Se trabajará desde una perspectiva proyeccionista del léxico (Alonso 2007; Bosque, 2004), específicamente desde las nociones de 'contorno lexicográfico' y 'restricciones de selección léxica', tal como las entiende la gramática moderna. Dicho de otro modo, los autores de esta investigación se proponen estudiar en qué medida los diccionarios de lengua española están proporcionando a los estudiantes datos útiles y suficientes, no solamente para descodificar significados, sino también -y en igual proporción- codificarlos.

${ }^{2}$ Educarchile "Error en el reconocimiento de vocabulario contextual", consultado el 11-dic2015. http://ww2.educarchile.cl/PORTAL.HERRAMIENTAS/psu/Resources/Pdfs_Tips/TIPS_ Lenguaje_2009/Tips4Lenguaje.pdf 
Con este fin, se pondrá a prueba la competencia de un grupo de 41 jóvenes chilenos, a quienes se aplicará un test de uso de léxico basado en definiciones provenientes de fuentes lexicográficas distintas, para comparar su grado de eficacia. Consideramos que una investigación de estas características podría ayudar a conocer el estado en que se encuentra el desarrollo de una de las competencias lingüísticas básicas de los jóvenes chilenos (la competencia 'codificadora'), en relación con las herramientas de que dispone la escuela para su aprendizaje; por otro lado, serviría para proponer nuevas formas de definición lexicográfica más idóneas para satisfacer las necesidades comunicativas del hombre moderno, que involucran no solo la facultad de 'comprensión' lingüística sino también la de 'producción'. En el segundo apartado, de marco teórico, se presentarán dos de los diccionarios de lengua española más importantes del último tiempo: DiCE (Diccionario de Colocaciones de Español (en proceso) y DAELE (Diccionario de Aprendizaje de Español Lengua Extranjera) (en proceso); en el tercer apartado se describirá la metodología de trabajo; en el cuarto se ofrecerá el análisis de los resultados; y en el quinto, las conclusiones.

\section{ESTADO DE LA CUESTIÓN}

Los diccionarios modernos de español presentan la particularidad de estar confeccionados con arreglo a las necesidades actuales de los individuos, razón por la cual incorporan cada vez más información útil para sus usuarios, de índole no solo semántica sino también léxico-combinatoria, sintáctica, morfológica, ortográfica, ortológica, etc. Es decir, la nueva lexicografía española, junto con hacer preferentemente diccionarios "de uso", persigue que estos sean fuentes valiosas de consulta para cualquier aprendiz del idioma, nativo o extranjero. Del sinnúmero de innovaciones que es dado comprobar en la lexicografía española contemporánea, destaca el tratamiento de las unidades léxicas predicativas, las que no suelen definirse ya (únicamente) mediante sinónimos, sino también de acuerdo con su estructura actancial, esto es, la red de argumentos que la misma entrada selecciona, y sin la cual esta no podría usarse en contextos reales de habla. Además, con la clara finalidad de hacer del diccionario de lengua un instrumento cada vez más "eficaz", a los repertorios tradicionales, semasiológicos y onomasiológicos, los lexicógrafos españoles han sumado formatos nuevos, como los de DiCE y DAELE³, ambos en proceso de elaboración. Nos referiremos a cada uno de estos diccionarios por separado.

${ }^{3}$ Ver siglario, al final. 


\subsection{DiCE, Diccionario de colocaciones del español}

Estrictamente hablando, este es el primer diccionario de colocaciones de español. El proyecto se encuentra todavía en curso, y es de consulta gratuita en la web. DiCE se ha propuesto recoger unidades léxicas predicativas en su macroestructura, con la salvedad de que estas son ahora nombres de sentimientos (afecto, animosidad, complacencia, desolación, gratitud, odio, etc.). Es interesante ver cómo en la presentación misma del lema (entrada o cabeza del artículo lexicográfico) el diccionario despliega la red de argumentos correspondientes (actantes, en la terminología del DiCE), cada uno de los cuales aparece representado mediante una letra (X, Y, Z, W, etc.).

Veamos el ejemplo de agradecimiento, primera acepción:

agradecimiento 1a m. (Sentimiento). "agradecimiento de individuo X por hecho $\mathrm{Y}$ a individuo Z".

En la teoría en que se enmarca este diccionario (teoría 'Sentido-Texto' de Mel'čuk $)^{4}$, los tres actantes de este predicado en la acepción indicada son:

1. $\mathrm{X}=$ el o los que sienten agradecimiento (por ejemplo, "agradecimiento del estudiante"),

2. $\mathrm{Y}=$ hecho, razón o motivo del agradecimiento (por ejemplo, "agradecimiento por condonar la deuda"),

3. $\mathrm{Z}=$ aquel o aquellos a quienes se dirige el agradecimiento (por ejemplo, "agradecimiento al ministro de Educación").

La totalidad de los actantes del nombre agradecimiento pueden estar explícitos en un solo $\mathrm{SN}$, en cualquier orden, como en "El agradecimiento a la diputada del partido $(\mathrm{Y})$ por su participación en la reunión ampliada de vecinos $(\mathrm{Z})$ de parte del alcalde de Santiago (X)". Nótese cómo los actantes del nombre agradecimiento son los mismos del verbo agradecer, del que deriva. En este último caso, es más fácil advertir que a cada actante corresponde una función sintáctica y otra se-

${ }^{4}$ El DiCE surgió en el marco de la Lexicología Explicativa y Combinatoria de Mel’čuk, dentro de cuyos presupuestos teóricos se han elaborado también el Dictionnaire explicatif et combinatoire du français contemporain (DEC, Mel'čuk et al. 1984-1999) y el Lexique actif du français (Mel'čuk y Polguère, 2007). Antes que el DiCE, fue el DEC el que utilizó formas proposicionales (del tipo X vendió $\mathrm{Y}$ a $\mathrm{Z}$ por $\mathrm{W}$ ) para presentar y definir entradas léxicas. He aquí un ejemplo para castigar: " $X$ castiga a $Y$ con (con /sin) $Z$ por W", cuya definición reza así: "X, que tiene poder sobre $Y$, teniendo la certeza de que $\mathrm{W}$ ha tenido lugar y que $\mathrm{Y}$ es el responsable de W, // X causa un daño a Y, con el fin de que la acción W de Y no se repita e Y sea consciente del carácter malo de W”. A nuestro juicio, más que una definición, esta es una explicación del definido. 
mántica: "El alcalde de Santiago (X-sujeto) AGRADECió a la diputada del partido (Y-complemento indirecto) por su participación en la reunión ampliada de vecinos (Z-complemento preposicional)", donde X es el agente de la acción de agradecer; Y, el destinatario de dicha acción y Z, el motivo por el cual aquella tiene lugar.

Conjuntamente con los actantes de la entrada léxica (nombre predicativo, en este caso), el DiCE se hace cargo de su régimen actancial y de su combinatoria léxica (esto es, de sus colocaciones). En cuanto al régimen, el diccionario nos ofrece un esquema en el que los distintos actantes aparecen vinculados, no ya a una función sintáctica, sino a una categoría sintáctica. Por ejemplo, para expresar la idea del "agradecimiento de X por Y a Z", la gramática del español cuenta con ciertos elementos lingüísticos y ciertos patrones combinatorios, restrictivos, que se activan en el discurso de los hablantes (de manera espontánea en los nativos), como puede observarse en esta tabla:

Tabla I. Esquema de régimen: agradecimiento de individuo $X$ por hecho $Y$ a individuo $Z$.

\begin{tabular}{|l|l|}
\hline Actantes & \multicolumn{1}{c|}{ Realizaciones } \\
\hline $1-\mathrm{X}$ & $\begin{array}{l}\text {-de N (prep de }+N(\text { ombre }) \\
\text { agradecimiento del presidente } \\
\text {-Apos (aposición) } \\
\text { mostró su agradecimiento con ese regalo }\end{array}$ \\
\hline \multirow{2}{*}{$\mathrm{Y}$} & $\begin{array}{l}\text {-por N (prep por }+N(\text { ombre) agradecimiento por las gestiones } \\
\text { realizadas } \\
\text {-por Vinf (prep por }+ \text { verbo en infinitivo) } \\
\text { agradecimiento por haber hecho el esfuerzo }\end{array}$ \\
\hline $3-\mathrm{Z}$ & $\begin{array}{l}\text {-a N (prep } a+N(\text { ombre) } \\
\text { agradecimiento a la diplomacia noruega } \\
\text {-hacia N (prep hacia }+N \text { (ombre) } \\
\text { agradecimiento hacia los voluntarios }\end{array}$ \\
\hline
\end{tabular}

Este esquema indica que la realización sintáctica de los actantes presenta alternancias; así, podemos hablar del "agradecimiento de alguien" o de "su agradecimiento" para referirnos al individuo que experimenta este sentimiento. La causa que provoca el agradecimiento se expresa a través de la preposición por $+\mathrm{SN}$ (agradecimiento por algo), o bien a través de por + cláusula con verbo en infinitivo (agradecimiento por haber hecho algo). Por último, para aludir al destinatario del agradecimiento podemos emplear el esquema: preposición $a+\mathrm{SN}$ (agradecimiento a alguien) o el de preposición hacia + SN (agradecimiento hacia alguien). 
En lo concerniente a las colocaciones, tratándose de predicados nominales, el diccionario recoge, entre otros elementos, los verbos y adjetivos con que los nombres aparecen normalmente combinados, formando sintagmas. Consideraremos en primer lugar las colocaciones formadas por "verbo + agradecimiento", y veremos cómo se expresan estas en el uso de los hablantes de la lengua española. Aquí revisaremos solo algunas combinaciones; por ejemplo, aquellas en las que los verbos sirven para expresar el sentido de que '[alguien] siente o experimenta agradecimiento [hacia algo o alguien] [por algo]':

\section{$[-]$ sentir - OPER 1 deber, sentir, tener, tributar} deber [(ART) - ]. Ejemplos:

1. Por condenarme a la vida, no te debo ningún agradecimiento

2. Otro ilustre preboste al que debemos eterna admiración y agradecimiento fue el técnico don Antonio Castilla sentir [ART - ]. Ejemplo:

1. Sintiendo el inmenso cariño y agradecimiento que te tengo tener [ART - ].Ejemplo:

1. Franco no tiene nada más que agradecimiento tributar $[\neg]$ Ejemplos:

1. Directores de compañías, poetas, críticos, periodistas y público tributan agradecimiento y honores $(\mathrm{R})$.

2. Yo personalmente le quiero tributar agradecimiento a Walter Ángel, que me acompañó toda la carrera, apoyando y alentando (web).

Los verbos destacados en cada sub-entrada (deber, sentir, tener, tributar) funcionan en la colocación como verbos operacionales (Oper); esto significa que sirven de soporte o de apoyo a la predicación propiamente tal (agradecimiento). El número después de Oper (Oper1) remite al primer actante del predicado (aquel que experimenta el sentimiento en cuestión). Si, en lugar del experimentante, quisiéramos referirnos al destinatario del agradecimiento, el verbo de apoyo funcionaría como Oper2 (por ejemplo, en "Ha sido el único agradecimiento que he tenido en mi vida”, DiCE, s.v.).

$\mathrm{Si}$ atendemos ahora a las colocaciones que pueden formarse con "agradecimiento + adjetivo", el listado que nos ofrece DiCE es el siguiente:

\section{[-] intenso Magn enorme, extremo, grande, infinito, sumo, total}

enorme Ejemplos y acudiendo ante Su Ilustrísima expresó a ésta su decidido propósito, aun manifestando su enorme agradecimiento a las numerosas pruebas de confianza recibidas, de recuperar su estado en la forma más elemental, de consagrarse al culto y al sagrado ministerio

extremo Ejemplos Éste llegó a querer a la pareja, la cual le demostraba 


\section{extremo agradecimiento.}

grande Ejemplos Cuando llego la hora de terminar la fiesta, todos sus amigos le dieron un apretón de manos con gran agradecimiento (web) infinito Ejemplos Por último, mi agradecimiento infinito a Pablo y a Marina por la paciencia y el cariño con el que me han dispensado horas que eran suyas por derecho (web). Eso era algo realmente especial y despertaba en Ana un agradecimiento infinito y la sensación de estar en deuda con esa persona (web)

sumo Ejemplos Me complazco en comunicarle que Su Santidad el Papa Juan Pablo II ha recibido con sumo agradecimiento la cantidad de ptas. 7.600.000 que Vuestra Excelencia ha tenido la gentileza de enviarle (web) total Ejemplos Asimismo, mi agradecimiento es total a M. Carmen Pérez Sierra, por su paciencia y espíritu crítico desarrollados en las discusiones y correcciones de los diferentes capítulos de esta obra. Aguardo sumiso su esperada y bienvenida demanda de unidades, con mi total agradecimiento anticipado.

[-] intenso y bueno Magn + Bon entrañable

entrañable Ejemplos Quisiera dejar constancia de que mi paso por el Ejército fue positivo, y guardo un entrañable agradecimiento por muchos de aquellos que anónimamente contribuyeron en esta fase de mi recuperación. que dura mucho Magn_temp eterno, imperecedero

eterno Ejemplos Otro ilustre preboste al que debemos eterna admiración y agradecimiento fue el técnico don Antonio Castilla. Con ello, don Jaime, se hace usted acreedor a mi eterno agradecimiento

imperecedero Ejemplos este querido $\mathrm{ABC}$, su presidente Nemesio Fernández-Cuesta, su director José Antonio Zarzalejos tienen mi agradecimiento imperecedero por haber publicado, hasta el día de la fecha, 211 artículos del plumilla que suscribe (web). Muchas personas han colaborado y colaboran de una u otra forma en este proyecto doctoral. A todas ellas mi imperecedero agradecimiento (web).

bueno Bon cálido Ejemplos Más bien siento un ligero, difuso pero cálido agradecimiento.

malo Anti Bon frío Ejemplos La petición es cálida, el agradecimiento es frío (web).

verdadero Ver sincero Ejemplos Los calurosos y larguísimos aplausos que le dedicó el público, que llenaba a rebosar el teatro de Montjuic, fueron de sincero agradecimiento y admiración.

Así como las colocaciones verbo-nominales anteriores, organizadas en torno al símbolo "Oper", las formadas por "agradecimiento + adjetivo" (o viceversa) también se ordenan aquí según ciertas pautas de sentido o "funciones léxicas", nombre 
que reciben en la teoría Sentido-Texto. La primera de estas es Magn, que sirve para cuantificar (o intensificar) lo denotado por el nombre (así, podemos decir que el agradecimiento que sentimos es enorme, infinito, total, etc.). Además de cuantificar los sentimientos, los hablantes podemos expresar su naturaleza, decir si son buenos (Bon), malos (Anti-Bon), efímeros o duraderos (temp), verdaderos (Ver) o fingidos (Anti-Ver), etc. Estas son algunas de las 60 funciones léxicas de validez lingüística universal que se han formulado en Lexicología Explicativa y Combinatoria (Mel'čuk y Polguère, 2007) y que se utilizan sistemáticamente en el DiCE para dar a conocer que, en las lenguas, la combinatoria léxica no es azarosa, sino que se fundamenta en cuestiones semánticas.

\subsection{DAELE, Diccionario de aprendizaje del español como lengua extranjera}

Es un diccionario de consulta online, también en proceso de elaboración. Está dirigido a estudiantes de nivel avanzado, y está pensado principalmente para la producción lingüística, al igual que DiCE. Por el momento consta de 363 entradas verbales, descritas integralmente (en términos semánticos, sintácticos, léxicos, morfológicos, ortográficos, pragmáticos y otros). Su mayor virtud es que usa la definición 'frástica', aquella que puso de moda el Cobuild ${ }^{5}$, y que se considera la más idónea "para ofrecer fácilmente la selección de argumentos y la construcción sintáctica, sin recurrir a formalismos", como los del DiCE (ver Presentación del diccionario). Se ha elegido la voz deslumbrar para ilustrar el modo en que DAELE describe y define sus entradas:

deslumbrar/se:

1 IMPEDIR transitivo Una luz deslumbra a alguien cuando le impide ver por ser intensa y estar dirigida a sus ojos:

La luz excesiva dirigida directamente a los ojos le puede deslumbrar.

deslumbrarse (con/por) pronominal (cambio de OD a sujeto) Alguien se deslumbra cuando pierde momentáneamente la visión a causa de una luz intensa y directa:

Se deslumbra con los reflejos del sol. (DAV)

2 PRODUCIR/ SENTIR ADMIRACIÓN transitivo Algo o alguien deslumbra a una persona cuando le produce una intensa admiración que puede impedirle ver sus defectos:

\footnotetext{
${ }^{5}$ Por ejemplo, en la primera acepción verbal de pay, este diccionario nos dice, entre otras cosas: "When you pay something such as a bill or a debt, you pay the amount that you owe". Una definición así formulada, muy próxima al uso de la pieza léxica, permite expresar el sujeto de pay (When you pay...), el objeto directo (pay something such as a bill or a debt) y el destinatario (pay to someone); es decir, sus tres argumentos básicos.
} 
Mi abuelo me deslumbraba con sus trucos de magia. (IULA)

[absoluto] La novela deslumbra por su originalidad. (DAV)

deslumbrarse (con/por/ante) pronominal (cambio de OD a sujeto) Alguien se deslumbra ante una persona o cosa cuando siente una intensa admiración por ella, que a veces le impide ver sus defectos:

Se deslumbraba con sus narraciones. (DAV)

FAMILIA deslumbramiento (sust.) FAMILIA deslumbrante (adj.).

Se ha prescindido de muchos de los ejemplos que incorpora este diccionario para dar cuenta de las alternancias sintácticas del definido, pero se ha conservado las cuatro acepciones fundamentales del verbo, que se pueden parafrasear así:

a. X (una luz) deslumbra a Y (a una persona)

b. Y (una persona) se deslumbra conl por X (una luz)

c. X (alguien, algo) deslumbra a Y (a una persona)

d. Y (una persona) se deslumbra con/ por Y (algo, alguien).

Según puede apreciarse, mientras a) y b) corresponden a usos primitivos de deslumbrar, c) y d) describen usos figurativos del verbo. Por otra parte, tratándose de un verbo causativo, deslumbrar admite construcciones transitivas (por ej., "La hermosura de la muchacha deslumbró al coronel") e intransitivas con se (por ej., "El coronel se deslumbró por (o con) la hermosura de la muchacha".

Del mismo modo que este diccionario tiene en cuenta las alternancias sintácticas de los predicados verbales para elaborar sus definiciones (cuando aquellas existen), jamás descuida anotar las preposiciones (una o más de una) que los verbos rigen y necesitan para la producción lingüística. Si bien es cierto que DAELE no recurre a formalismos, sus definiciones no siempre son sencillas y, más que ayudar al usuario, a veces pueden confundirlo. Por otro lado, creemos que las etiquetas semánticas (IMPEDIR VER; PRODUCIR/ SENTIR ADMIRACIÓN) tienen mucha notoriedad en el artículo, en comparación con las entradas, objetos de análisis. Sin embargo, a nuestro parecer, la originalidad de la descripción lexicográfica (el uso de la definición-frase, la separación cuidadosa de las acepciones, la variedad de los ejemplos, etc.), es una muestra más de cómo los diccionarios contemporáneos de español están complejizando su microestructura con el objeto de poner de relieve el comportamiento gramatical de las piezas del lexicón, en el entendido de que la gramática y el diccionario no están nunca disociados en las lenguas naturales. 


\subsection{Problemas en la delimitación del contorno}

No obstante los significativos avances reseñados, los diccionarios actuales de español, particularmente los semasiológicos, persisten en ciertas falencias que es preciso superar. En primer lugar, datos “claves" para comprender y usar el definido aparecen señalados en las notas explicativas o en los ejemplos, es decir, fuera de las definiciones propiamente tales, que es donde deberían estar; en segundo lugar, en un mismo artículo lexicográfico, o bien en un mismo repertorio lexicográfico, las definiciones de unidades predicativas a veces expresan los contornos de los definidos y a veces no; en tercer lugar, no se ha uniformado convenientemente aún el modo en que los contornos habrían de expresarse dentro del mismo texto de la definición; en cuarto lugar, por la forma en que se recogen algunos contornos, no hay posibilidad de indicar si la entrada posee o no posee régimen preposicional, ni cuál es este exactamente, de poseerlo.

Tomemos como ejemplo el DLE. Para la entrada desabrido anota cuatro acepciones, para tres de cuyas definiciones incorpora un contorno claro, que expone al comienzo mediante la fórmula "Dicho de...":

desabrido, da

1. adj. Dicho de una fruta o de otro alimento: Que carece de gusto, o apenas lo tiene, o lo tiene malo.

2. adj. Dicho de una ballesta o de un arma de fuego, como la escopeta: Que es fuerte y dura al disparar, de manera que da coz o golpe al tirador.

3. adj. Dicho del tiempo: Destemplado, desigual.

4. adj. Áspero y desapacible en el trato (los sombreados son nuestros).

Lo que en cada caso contiene la fórmula introductoria son las restricciones léxicas de desabrido: el adjetivo se predica distintamente de 1) frutas o alimentos; 2) ballestas o armas de fuego; 3) tiempo atmosférico ${ }^{6}$. Nótese, sin embargo, que no se explicita contorno alguno en la definición; 4) "Áspero y desapacible en el trato", a pesar de que la palabra lo tiene en esta acepción y que gracias a él podemos entender su sentido. Clave, en cambio, lo incorpora debidamente (ver acepción 3):

desabrido, da

adj.1 Referido a un alimento, esp. a la fruta, con poco o ningún sabor, o con sabor desagradable: Esas fresas desabridas no me gustan ni con azúcar. 2 Referido al tiempo atmosférico, con variaciones desagradables: El mes de febrero tuvimos

${ }^{6}$ Las restricciones de 1) y de 2) muestran cómo, de significar 'particulares' en español, esta palabra pasó a significar también 'especies'. 
un tiempo muy desabrido. 3 Referido a una persona o a su carácter, que son desagradables o ásperos en el trato: Tiene un carácter antipático y desabrido.

Por su parte, $\mathrm{DEA}^{7}$ hace constar el contorno en una nota explicativa, pudiendo haberlo hecho en la misma definición, siguiendo el modelo de la acepción número $\operatorname{dos}^{8}:$

desabrido -da adj 2 [Comida, esp. fruta] insípida o de sabor desagradable. 3 Áspero o desagradable. Frec referido a pers o a su carácter o actitud.

La inexistencia de patrones comunes de definición se hace más evidente en este otro caso:

afable adj. inv. Agradable, afectuoso y amable en el trato y en la conversación con los demás: Es tan afable que hace sentirse a gusto a cualquie$\mathrm{ra}$, aunque sea un desconocido. (Clave)

afable 1. adj. Agradable, dulce, suave en la conversación y el trato. (DLE) afable adjetivo 1 (ser / estar) Que trata a los demás con agrado, amabilidad o afecto: No sé contigo, pero con nosotras estuvo muy afable. (DSAL) ${ }^{9}$

afable adj [Pers.] de trato amable o afectuoso. b) Propio de la pers. afable. No sé contigo, pero con nosotras estuvo muy afable. Es muy afable en el trato con los amigos. Nuestra editora es muy afable. (DEA).

Clave y DLE definen mediante sinónimos (afable: 'agradable, afectuoso, amable'). El primer diccionario proporciona al menos un ejemplo; el segundo, ninguno. DSAL, fuera de indicar que el adjetivo admite posición pre y postnominal, empieza su definición con "Que...", cuyo uso normalmente encontramos en los adjetivos relativos, aunque aquí pudiera ser una estrategia para expresar que, en tanto adjetivo, afable se predica de un nombre (un sustantivo) implícito en la definición y que el lector no tiene dificultad en identificar, por el contexto que crea la definición misma (así también en los casos de Clave y DLE). DEA, por último, hace constar dicho nombre precisamente en el contorno del definido, entre corchetes: [Pers.].

Tan grave como lo anterior nos parece que los diccionarios no ofrezcan pistas a los lectores sobre la construcción sintáctica de las entradas léxicas. Compárense

${ }^{7}$ Diccionario del Español Actual

${ }^{8}$ La definición de la acepción 3 de desabrido podría haber quedado así, poco más o menos: desabrido,-da adj 2 [Comida, esp. fruta] insípida o de sabor desagradable. 3 [Persona, esp. su carácter o sus actitudes] Áspero o desagradable.

${ }^{9}$ Diccionario Salamanca de lengua española. 
las siguientes definiciones de asiduo:

asiduo, dua 1. adj. Frecuente, puntual, perseverante. (DLE)

asiduo, dua adj. Frecuente o constante: Se ha convertido en un espectador asiduo a todas nuestras funciones. (Clave)

asiduo -dua adj 1 [Cosa] que se hace con constancia y continuidad. 2 [Pers.] que realiza con constancia y continuidad una acción determinada. b) [Pers.] que acude con frecuencia y constancia [a un lugar (compl de posesión)] Tb sin compl, por consabido. Tb n. (DEA).

Tal como se aprecia en las citas, solo DEA, en 2 b), nos advierte de que asiduo tiene dos argumentos (primeramente el sustantivo del que se predica; luego, su complemento). Esta misma información podría deducirse del ejemplo que nos presenta Clave, "...espectador asiduo a todas nuestras funciones", pero nos encontramos con que el régimen preposicional es distinto en cada diccionario. DEA parece indicarnos que, en lugar de "asiduo a", la construcción es "asiduo de". ¿O es que este adjetivo acepta ambas preposiciones? El diccionario Larousse (2012) señala en el ejemplo que tal preposición es de:

asiduo, a adj./ s. Que asiste a un lugar o realiza cierta actividad con frecuencia. EJEMPLO: es un asiduo del club, viene cada día ${ }^{10}$.

Según nuestro parecer, si en el uso se constata que cierto adjetivo no presenta un régimen único, las alternancias preposicionales tendrían que quedar expresadas claramente, pero no solo en los ejemplos del artículo lexicográfico. Para lograr esto, nosotros hemos propuesto el siguiente diseño:

asiduo, a $[\mathrm{X}(\mathrm{es})-a / d e \mathrm{Y}]$ Dicho de una persona, que asiste regularmente a un sitio o realiza ciertas actividades con frecuencia. Luis es asiduo lector de El Mercurio; Su esposa es donante asidua de la Cruz Roja; He visto muchas mujeres asiduas al estadio en estos tiempos.

En primer lugar, nos parece útil emplear una 'forma proposicional' semejante a la del DEC y la del DiCE, que hemos ubicado después del lema y antes de la definición, como en asiduo (vide supra) [zona sombreada, entre corchetes], donde $\mathrm{X}$ representa al argumento 1 del adjetivo (el sustantivo) e $\mathrm{Y}$ representa al argumento 2 (el complemento del adjetivo); la virgulilla $(\sim)$ está en lugar del lema; el

${ }^{10}$ El CREA (Corpus de Referencia del Español Actual, de la Real Academia Española, consultado el 6 de julio de 2016) arroja concordancias con $a$ y de, indistintamente: "Carlos Arce que es asiduo a esta sección y vive en Porto Alegre...”; “... esta vez no pone el apellido pero es cliente asiduo de la sección..." 
verbo (ser) entre paréntesis indica que asiduo puede formar parte de una oración atributiva ("Mi padre fue asiduo a las carreras de caballos") o no atributiva ("En la actualidad hay poca gente asidua a las fiestas de toros"); la o las preposiciones regidas aparecen en cursiva, separadas por barra oblicua ( / ) si hay más de una. En cuanto a los ejemplos que se utilicen en el artículo para ilustrar la construcción sintáctica de la pieza definida, se pensó que es mejor que sean creados ad hoc, de modo de poder replicar la distribución de los elementos lingüísticos ya expuestos en la forma proposicional.

Para terminar este apartado, queremos añadir que nos parece imprescindible que las definiciones de los adjetivos comiencen por la fórmula "Dicho de..." (o "Referido a...") porque todos ellos son unidades dependientes sintácticamente de un sustantivo. De todos los diccionarios semasiológicos citados hasta el momento, DLE y Clave no han desterrado totalmente de su práctica lexicográfica la (mal) llamada definición sinonímica ${ }^{11}$, cuyo mayor inconveniente es que normalmente los elementos que se usan para definir (aquí, adjetivos) no tienen la misma selección léxica que los definidos.

En lo que sigue, se intentará aplicar la propuesta que acabamos de explicar a tres adjetivos de la lengua española, para comprobar su eficacia. Enseguida, se mostrará cuáles fueron los esquemas definicionales utilizados para cada uno de los adjetivos seleccionados, así como también, la manera en la que se llevó a cabo el proceso de selección de la muestra y de aplicación de nuestro instrumento.

\section{METODOLOGÍA}

\subsection{Hipótesis}

La hipótesis de este estudio sostiene que, si el proceso de descodificación para los educandos chilenos resulta deficiente -debido a que no se ha desarrollado correctamente en la escolaridad-, probablemente esto afecte de forma directa su capacidad de codificación. Por ello, a través de la utilización de dos tests, pretendemos comprobar cómo, entregando información semántica más precisa en la entrada lexicográfica de un predicado (adjetivo y verbo particularmente), se puede lograr una mejor descodificación y, junto con ello, una codificación más exitosa.

${ }^{11}$ Lamentablemente, Clave suele inspirarse mucho en DLE para elaborar sus propias definiciones. Compárense las definiciones de suculento, pleno y pingüe en ambos diccionarios:

DLE suculento adj. Sabroso, jugoso o nutritivo; pleno adj. Lleno o completo; pingüe adj.inv. abundante, copioso o fértil.

Clave suculento adj. Jugoso, sustancioso, exquisito; pleno adj. Completo, lleno; pingüe. adj. abundante, copioso, fértil. 


\subsection{Participantes}

Para llevar a cabo la comprobación de nuestra hipótesis se evaluó a un total de 42 jóvenes (damas y varones) de un establecimiento educacional técnico-profesional (particular subvencionado) de la comuna de Teno en la Provincia de Curicó, VII Región del Maule. De los 42 sujetos, 21 pertenecían al curso de Enfermería (especialidad 'atención de enfermos'), mientras que los 21 jóvenes restantes pertenecían al curso Agrícola (especialidad 'agropecuaria') del establecimiento. El rango de edad de nuestros informantes, de tercer año de educación media, oscilaba entre los 16 y 17 años.

\subsection{Variables y diseño}

Para efectos de este estudio se ha adoptado un enfoque de investigación mixto y de alcance exploratorio, toda vez que está orientado hacia el análisis de la capacidad codificadora de estudiantes del primer ciclo de enseñanza media desde una perspectiva léxico-sintáctica, que ha sido poco o nada abordada en educación.

Los estudiantes que participaron de este estudio fueron escogidos de acuerdo con un único criterio de selección: el "adecuado" nivel de comprensión de lectura (Centro educacional Aquelarre: 96,9\%) con el que fueron evaluados por la prueba SIMCE 2014 para alumnos de $2^{\circ}$ nivel de enseñanza media ${ }^{12}$, aquella que nuestros informantes tuvieron la obligación de rendir el año anterior.

De este modo, nos cercioramos de que la muestra estuviera conformada por individuos competentes en el dominio lingüístico que este estudio se propuso observar.

\subsection{Instrumentos}

El corpus de la investigación fue elicitado mediante dos instrumentos de evaluación diseñados por los autores de este estudio, conforme a los cuales los participantes debían crear oraciones, o ejemplos de uso para tres adjetivos del español: Adusto(A), Engorroso(A) e Inefable, los que fueron seleccionados por tratarse de léxico poco frecuente en el vocabulario de los jóvenes. A nuestro parecer, dicha situación propiciaría una lectura más detenida de la información lexicográfica por parte de los informantes, lo que les permitiría asimismo decodificar la información

${ }^{12}$ En el siguiente enlace se pueden conocer más detalles sobre el balance que realizó el MINEDUC con respecto a los resultados obtenidos por este establecimiento educacional en la prueba SIMCE 2014. http://www.simce.cl/ficha/?rbd=16477 
de modo más objetivo y, posteriormente, lograr una codificación menos influenciada por los usos cotidianos y conocidos.

Los dos instrumentos se diferenciaban en el tipo de definiciones que aportaban para cada una de las unidades léxicas. Para el Test 1, las definiciones fueron tomadas del Diccionario de la lengua española ${ }^{13}$, DLE (2014), mientras que para el Test 2 fueron creadas por nosotros a partir de una propuesta de definición lexicográfica cuya virtud es explicitar los contornos y las restricciones léxicas que forman parte del contenido semántico-sintáctico de las entradas del diccionario ${ }^{14}$. De este modo, las definiciones que se ofrecieron a los alumnos en cada uno de los tests fueron las siguientes:

Tabla II. Definiciones lexicográficas proporcionadas para los adjetivos seleccionados en el Test 1 y Test 2.

\begin{tabular}{|l|l|}
\hline \multicolumn{1}{|c|}{$\begin{array}{l}\text { DLE (2014) } \\
\text { Test 1 }\end{array}$} & \multicolumn{1}{c|}{$\begin{array}{c}\text { Serra (2013) } \\
\text { Test 2 }\end{array}$} \\
\hline $\begin{array}{l}\text { adusto, ta. Poco tratable, huraño, mal- } \\
\text { humorado. }\end{array}$ & $\begin{array}{l}\text { adusto, a [X (es) -] Dicho de una per- } \\
\text { sona, de su carácter, gestos o adema- } \\
\text { nes, que es huraña, muy seria y nada } \\
\text { amable. }\end{array}$ \\
\hline engorroso, sa. Dificultoso, molesto. & $\begin{array}{l}\text { engorroso, a [X (es) - Dicho de un } \\
\text { asunto, un problema o un trámite, que } \\
\text { es complejo, lento y difícil de resolver. }\end{array}$ \\
\hline $\begin{array}{l}\text { inefable. Que no se puede explicar con } \\
\text { palabras. }\end{array}$ & $\begin{array}{l}\text { inefable. [X (es) - Dicho de senti- } \\
\text { mientos o emociones muy intensos, } \\
\text { que no pueden ser descritos o explica- } \\
\text { dos con palabras. }\end{array}$ \\
\hline
\end{tabular}

\subsection{Procedimiento}

Inicialmente, se decidió que cada grupo respondiera consecutivamente ambos tests, pero luego se pensó en la posibilidad de que las respuestas pudieran con-

${ }^{13}$ Para crear nuestras propias definiciones nos servimos de la información proporcionada por tres importantes fuentes lexicográficas: Clave (2003), DSAL (2007), y DLE (2014). La decisión de poner a prueba las definiciones del DLE se debió al hecho de que la mayor parte de los repertorios lexicográficos vigentes de español (aquí y en España) basan sus contenidos en el diccionario académico. Por otro lado, en las escuelas chilenas, así como en los medios de comunicación, este diccionario representa la autoridad máxima en materia de lenguaje.

${ }^{14}$ Cf. Serra (2013). 
taminarse -por la proximidad de las tareas-, así que, finalmente, se aplicó de modo paralelo un test distinto a cada grupo. El Test 1 correspondió al curso de Enfermería, que debió crear oraciones según las definiciones del DLE (2014) para los tres adjetivos seleccionados. El Test 2 correspondió al curso de Agropecuaria, que debió hacer oraciones para los mismos adjetivos, pero cuyas definiciones se formularon de acuerdo con la propuesta de Serra (2013). Este procedimiento se llevó a cabo en ambos cursos en un plazo de aproximadamente 15 a 20 minutos, sin que haya presentado dudas o complicaciones a los alumnos.

\section{ANÁLISIS DE RESULTADOS}

Los resultados que se obtuvieron serán expuestos, en esta sección, en tres apartados distintos. Cada una de estos estará dedicado a comentar, comparativamente, los resultados de ambos tests para cada adjetivo, vale decir, para Adusto(A), ENGORROSO(A) e INEFABLE.

\subsection{Adusto(a)}

Según la información que nos proporciona el diccionario académico, para esta palabra existen tres acepciones; de estas se escogió solo la segunda, por parecernos que las otras son desconocidas (o de uso muy literario) en el español de Chile.

\section{adusto, a}

1. adj. Quemado, tostado, ardiente.

2. adj. Poco tratable, huraño, malhumorado.

3. adj. Seco, severo, desabrido. Paisaje adusto. Prosa adusta.

De acuerdo con el total de respuestas obtenidas mediante la aplicación de ambas pruebas de vocabulario, se puede decir que las oraciones generadas por nuestros informantes no revelaron diferencias significativas, en términos comparativos, ya que de los 42 textos elicitados para esta palabra, solo 3 (el 14,3\%) presentaron casos de agramaticalidad: 


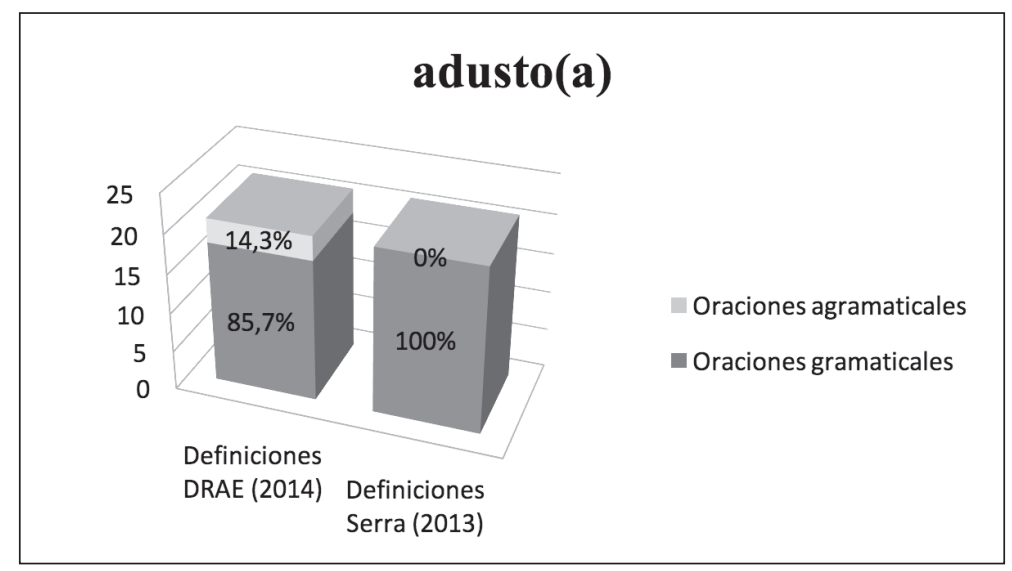

Gráfico 1. Resultados arrojados por el adjetivo adusto(a).

Oraciones agramaticales:

\section{Test 1: Definiciones DLE (2014)}

No 1: Un día cualquiera el perro se encontraba muy adusto.

No 4: Mi gato es adusto cuando lo despiertas.

No 8: Nosotros los estudiantes estamos realmente con una realidad adusta por parte de estado.

Como se aprecia en el Gráfico 1, dichas oraciones agramaticales fueron generadas solamente a partir de la definición del DLE (2014), la que, contrariamente a la nuestra, no incorpora debidamente las restricciones semánticas contenidas en la entrada (el adjetivo adusto/a). De este modo, se explica por qué en el test 1 aparecen construcciones irregulares como la No 1 y la No 4, donde la idea de que algo es adusto no se dice de una persona, de sus gestos o de su carácter (como proponemos nosotros), sino que se utiliza para decir de un animal e incluso de algo tan abstracto como "la realidad", según muestra la oración No 8. En el caso de las oraciones restantes -18 obtenidas del Test $1(85,7 \%)$ y 21 del Test $2(100 \%)-$, no se presentan irregularidades de orden gramatical, por cuanto los alumnos tienen en cuenta las restricciones léxicas correspondientes a la entrada, tal como nos indican los siguientes ejemplos:

Test 1: Definiciones DLE (2014)

No 6: Mi madre es una persona muy adusta.

No 10: La señora del registro civil es adusta.

No 12: Mi compañero es adusto.

No 18: Los viejos pueden ser muy adustos a veces. 


\section{Test 2: Definiciones Serra (2013)}

No 23: No me pareció tan buena esta charla, ya que la persona que la presentó tuvo una actitud sumamente adusta.

No 24: Tiene un carácter adusto y es difícil de tratar

No 31: Ayer en la ventanilla del banco me atendió un hombre muy adusto.

No 37: Ese hombre enojón hizo un gesto adusto.

No 40: La Javiera cuando se molesta es adusta.

No 42: Su expresión facial es un poco adusta.

Si bien en términos generales se puede decir que en su totalidad estas oraciones están bien construidas, destaca el hecho de que, en aquellas generadas a partir de las definiciones del DLE (2014), el adjetivo aparece asociado de modo general al clasema de 'persona' (ver casos No 6, No 10 , No 12 y No 18), mientras que en el caso de las elicitaciones producidas a partir de las definiciones de Serra (2013), adusto también se aplica a los gestos, a la actitud o al carácter de las personas, como puede observarse en las oraciones No 23 , No 24 y No 37 , No 42, respectivamente. A esto se suma el hecho de que las oraciones del Test 2 la mayoría de las veces presentaron elementos adicionales que enriquecieron el nivel de expresividad con el que se manifestaron los jóvenes, en comparación con las del Test 1, más simples (elementales) y escuetas. Estos datos adicionales, que aparecen sombreados en los ejemplos de más arriba, suponemos que han sido usados en la creación de oraciones por tratarse de información que hemos proporcionado en nuestra definición de adusto(a). Así, se explica por qué, por ejemplo, Javiera es adusta "cuando se molesta", o por qué un carácter es adusto cuando "es difícil de tratar". De este modo, las oraciones hechas en base a nuestro modelo suelen incorporar nuevas notas semánticas, en consonancia con el sentido de que alguien es adusto cuando es 'huraño, muy serio y nada amable' en el trato con los demás.

\subsection{Engorroso(a)}

Los resultados obtenidos para engorroso(a) son bastante más significativos que los de la entrada anterior. Para este adjetivo se generaron 42 oraciones en total, de las cuales 15 presentaron algún tipo de agramaticalidad. Lo interesante de este caso queda a la vista en el Gráfico 2, ya que las 15 oraciones agramaticales de las que hablamos $(71,4 \%)$ corresponden al Test 1 , de modo que las definiciones del DLE (2014) esta vez propiciaron un margen de error más amplio, donde solo 6 oraciones $(28,6)$ fueron construidas correctamente. 


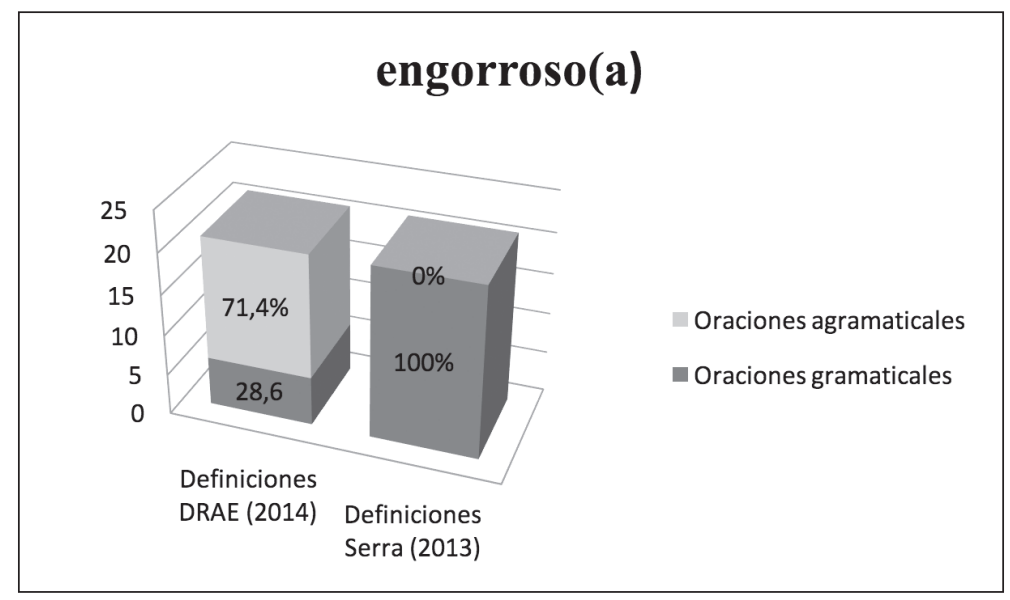

Gráfico 2. Resultados para el adjetivo engorroso(a).

Algunas de las oraciones agramaticales que se encontró fueron las siguientes:

Test 1: Definiciones DLE (2014)

No 3: El niño del segundo $C$ es muy engorroso.

No 6: Trato de ser una persona no engorrosa.

No 7: Esa habitación es engorrosa.

No 9: El ruido transmitido desde el televisor suele ser bastante engorroso.

No 10: El pantalón es engorroso para mi piel.

No 12: La música cumbia es engorrosa.

No 14: El perro de mi vecina es muy engorroso.

No 20: Que la gente me pase a llevar en la calle me hacer sentir muy engorrosa.

Como se puede ver, además de ser más amplio el margen de error que presentaron las oraciones creadas en base a las definiciones del DLE (2014), también son muy variadas las causas de dichas agramaticalidades. Si se tiene en cuenta que el adjetivo en cuestión se predica en realidad solo de "un asunto, problema o trámite que es complejo o difícil de resolver", nos percataremos de que los estudiantes que respondieron el Test 1 , al no disponer de esta información "crucial" para aprehender el significado de engorroso(a), usaron el adjetivo erróneamente para referirse a personas, animales, cosas u objetos, e incluso, a sentimientos. Ahora bien, estimamos que la definición de engorroso(a) ofrecida por el DLE no solo es deficiente por no señalar las restricciones léxicas inherentes a la entrada, sino que, además, es incorrecta porque señala como sinónimos suyos adjetivos que no son equivalentes semánticos necesarios y que, por lo mismo, confunden al lector ingenuo del diccionario. Se comprende, así, que una definición 'mal' diseñada pueda afectar 
la capacidad codificadora de los informantes, algunos de los cuales (ver ejemplos No 10 y No 12) emplean engorroso(a) con el mismo significado de 'molesto' para referirse a un pantalón y a un tipo de música, que, si bien pueden llegar a resultar molestos, no por ello son engorrosos.

Por el contrario, los resultados obtenidos en el Test 2 para este mismo adjetivo fueron totalmente positivos. Dicho de otro modo, el uso del adjetivo engorroso(a) en estas oraciones presentó una adecuada delimitación de las restricciones léxicas y se aplicó a 'un asunto', un problema o un trámite, tal como muestran los próximos ejemplos:

\section{Test 2: Definiciones Serra (2013)}

No 25: Sacar carnet es muy engorroso.

No 26: Mi papá quiere cambiar de color su auto y para eso debe hacer un trámite muy engorroso.

No 31: Sacar licencia es un trámite muy engorroso.

No 32: Juan debe ir al banco a resolver un problema muy engorroso.

\subsection{Inefable}

Finalmente, para el adjetivo inefable se han producido resultados tan reveladores como los examinados en el caso anterior. Para este tercer adjetivo se generaron 42 oraciones en total, de las cuales 16 resultaron ser agramaticales.

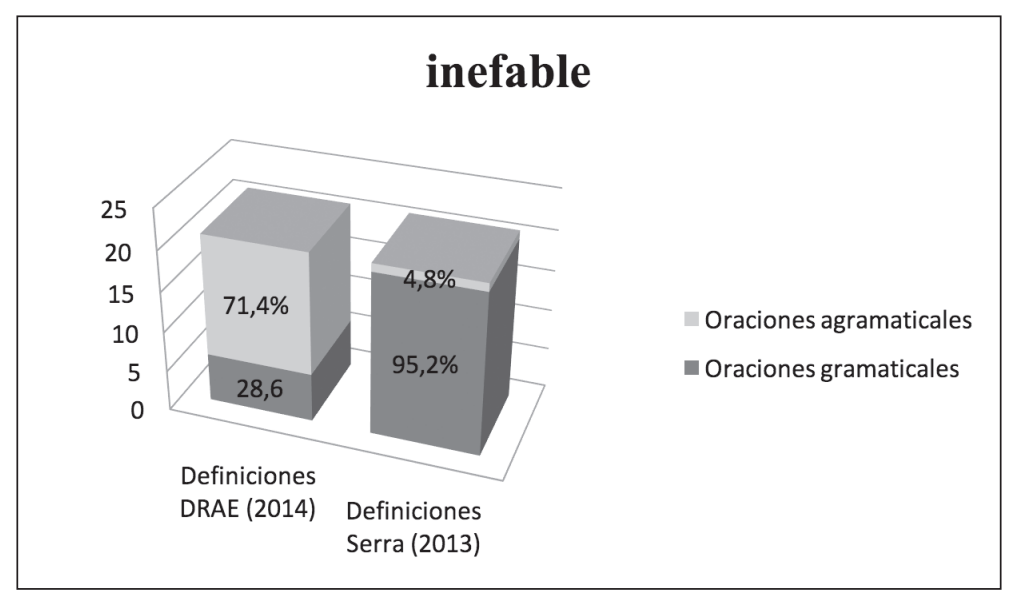

Gráfico 3. Resultados para el adjetivo inefable. 
Según el Gráfico 3, de dichas construcciones irregulares solo 1, es decir el $4,8 \%$, fue generada a partir de nuestra definición de inefable, mientras que las 15 oraciones restantes, que equivalen al $71,4 \%$, se obtuvieron a partir de la definición proporcionada por el DLE (2014). He aquí algunas de ellas:

\section{Test 1: Definiciones DLE (2014)}

No 1: Tus sopaipillas son inefables.

No 2: Ella es totalmente inefable.

No 6: En mi familia hay muchas cosas inefables.

No 8: Es inefable la contaminación ambiental.

No 18: Lo que está ocurriendo en Chile es inefable.

No 21: La película que vi en el cine es inefable.

Test 2: Definiciones Serra (2013)

No 28: El Michel es muy inefable, no entiende que todo se acabó.

Las 20 oraciones gramaticales producidas a partir de nuestras definiciones, nos revelan que al parecer los jóvenes comprendieron cuáles eran las restricciones léxicas adecuadas para utilizar el adjetivo inefable, el cual apareció (con excepción del caso No 28 que revisamos más arriba) aplicado a sentimientos en general, pero también a tristeza, pena, alegría, cariño, amor, etc., en particular, en perfecta consonancia con los rasgos semánticos destacados en la definición: "Dicho de sentimientos o emociones muy intensos, que no pueden ser descritos o explicados con palabras".

\section{CONCLUSIONES}

De acuerdo con los resultados obtenidos en la aplicación de nuestros instrumentos de evaluación, se puede concluir que las propuestas de definición del DLE (2014) para los adjetivos adusto(a), engorroso(a) e inefable, la mayoría de las veces generaron construcciones agramaticales por parte de nuestros informantes. Estas definiciones, en comparación con las nuestras, al prescindir de los contornos requeridos por la semántica de las respectivas entradas, favorecieron la agramaticalidad de las oraciones producidas por los jóvenes. En segundo lugar, y a diferencia de lo anterior, las definiciones formuladas por nosotros, mediante la propuesta de Serra (2013), dieron lugar casi siempre a oraciones gramaticalmente correctas, debido justamente al hecho de incorporar en el texto del definiens los semas correspondientes al definiendum.

Creemos, de este modo, que estamos en condiciones de corroborar nuestra hipótesis inicial, en el sentido de que los datos obtenidos demuestran que al ser deficiente la información desde la que los alumnos descodifican, suelen obtenerse 
codificaciones igualmente deficientes, de modo que si los alumnos durante sus años de escolaridad no tienen acceso a herramientas lingüísticas (en este caso diccionarios) aptas para el desarrollo de sus capacidades decodificadoras, probablemente en el futuro seguirán cometiendo errores al momento de emplear ciertas palabras en determinados contextos.

Por los motivos señalados nos parece que resultaría muy valioso un análisis crítico y minucioso de los errores que se cometen tanto en el proceso de elaboración de instrumentos lexicográficos como en el proceso de utilización de los mismos en la sala de clases. De igual modo, un estudio de estas características podría ser relevante si se considera que la dimensión léxico-sintáctica de las lenguas no solo tiene alcance en la etapa temprana de aprendizaje, sino que también en etapas posteriores, como cuando un estudiante desea aprender una lengua segunda o extranjera, o un hablante adulto desea aprender a utilizar de forma más eficiente su lengua nativa.

\section{REFERENCIAS}

Alonso Ramos, Margarita. (2007). Actantes y colocaciones. Nueva Revista de Filología Hispánica, LV, 2, 435-458.

Bosque, Ignacio. (2004). Combinatoria y significación. Algunas reflexiones. En I. Bosque (dir.) Redes. Diccionario combinatorio del español contemporáneo, lxxviiclxxi Madrid: SM.

Mel'čuk, Igor et al. 1984-1999. Dictionnaire explicatif et combinatoire du français contemporain. Recherches lexico-sémantiques. Montréal: Les Presses de l'Université de Montréal, I-IV.

Mel'čuk, Igor y Polguère, Alain. (2007). Lexique actif du français. L'apprentissage du vocabulaire fondé sur 20000 dérivations sémantiques et collocations du français, collection «Champs linguistiques». Louvain-la-Neuve: De Boeck Duculot.

Serra, Susana. (2013). Propuesta de definición lexicográfica para verbos y adjetivos del español. Revista de Lingüística Teórica y Aplicada, 51, 1, 71-100.

\section{Diccionarios/Repertorios}

CREA. Corpus de Referencia del Español Actual. Disponible en www.rae.es. Clave (2003). Clave. Diccionario de uso del español actual. Madrid: SM. Disponible en http://clave.smdiccionarios.com/app.php

Cobuild (1987). English Language Dictionary. UK: Collins.

DAELE. (en proceso). Diccionario de Aprendizaje del Español como Lengua Extranjera. Disponible en http://www.iula.upf.edu/rec/daele/ 
DEA. (1999). Diccionario del español actual. Madrid: Aguilar.

DEC. Dictionnaire explicatif et combinatoire du français contemporain, Recherches lexico-sémantiques. Vol. 1 (1984), vol. 2 (1988), vol. 3 (1992), vol. 4 (1999). Montreal: Les Presses de L’Université de Montréal.

DiCE. (en proceso). Diccionario de Colocaciones del español. Disponible en http://www.dicesp.com/consultageneral/lemas

DLE. (2014). Diccionario de la lengua española. (23 $3^{\text {era }}$ edic.). Disponible en http://dle.rae.es/

DSAL. (2007). Diccionario Salamanca de la lengua española. Madrid: Santillana y Universidad de Salamanca. Disponible en http://fenix.cnice.mec.es/diccionario/

Larousse. (2012). Diccionario avanzado de español. México: Ediciones Larousse. 